\title{
Worse disease-free, tumor-specific, and overall survival in surgically-resected lung adenocarcinoma patients with $A L K$ rearrangement
}

\author{
Qiongqiong Gao ${ }^{1,2,3,4}$, Pupu Li $^{1,2,3,4}$, Xiangli Jiang ${ }^{1,2,3,4}$, Zhongli Zhan ${ }^{1,2,3,5}$, Qingna \\ Yan ${ }^{1,2,3,5}$, Bo Zhang ${ }^{6}$ and Chun Huang ${ }^{1,2,3,4}$ \\ ${ }^{1}$ Tianjin Medical University Cancer Institute \& Hospital, National Clinical Research Center for Cancer, Tianjin 300060, P.R. \\ China \\ ${ }^{2}$ Key Laboratory of Cancer Prevention and Therapy, Tianjin 300060, P.R. China \\ ${ }^{3}$ Tianjin's Clinical Research Center for Cancer, Tianjin 300060, P.R. China \\ ${ }^{4}$ Department of Thoracic Oncology, Tianjin Cancer Institute \& Hospital, Tianjin 300060, P.R. China \\ ${ }^{5}$ Department of Pathology, Tianjin Lung Cancer Center, Tianjin Cancer Institute \& Hospital, Tianjin 300060, P.R. China \\ ${ }^{6}$ Department of Ultrasound Diagnosis, Second Hospital of Tianjin Medical University, Tianjin 300060, P.R. China \\ Correspondence to: Chun Huang, email: yellowpure@126.com \\ Keywords: anaplastic lymphoma kinase rearrangement, lung adenocarcinoma, surgical resection, clinicopathological \\ characteristics, treatment outcome \\ Received: March 17, $2017 \quad$ Accepted: August 26, $2017 \quad$ Published: September 18, 2017 \\ Copyright: Gao et al. This is an open-access article distributed under the terms of the Creative Commons Attribution License 3.0 \\ (CC BY 3.0), which permits unrestricted use, distribution, and reproduction in any medium, provided the original author and source \\ are credited.
}

\section{ABSTRACT}

Introduction: This study determined the prevalence of anaplastic lymphoma kinase $(A L K)$ rearrangement, and identified the associations of $A L K$ rearrangement with clinicopathologic characteristics and treatment outcomes in patients with surgically-resected stage I-III lung adenocarcinoma.

Methods: A total of $\mathbf{5 3 4}$ surgically-resected lung adenocarcinoma patients were studied. The prevalence of ALK protein over-expression was determined by a fullyautomated immunochemistry assay (with mouse monoclonal Ventana D5F3 antibody), and the associations of $A L K$ rearrangement with clinicopathologic characteristics and treatment outcomes were analyzed.

Results: Forty-two (7.9\%) of the 534 lung adenocarcinoma patients were $\boldsymbol{A L K}$ IHC-positive. ALK rearrangement was significantly associated with younger age $(P=0.011)$, high $\mathrm{T}$-stage $(P=0.025)$, high pathologic stage $(P=0.002)$, solid predominant adenocarcinoma with mucin production $(P=0.006)$, invasive mucinous adenocarcinoma $(P=0.009)$, and receipt of adjuvant therapy after surgery $(P=$ 0.036 ), but no significant associations were found between the $A L K$ rearrangement and sex or smoking status. ALK IHC-positivity was significantly associated with a shorter disease-free survival, tumor-specific survival, and overall survival $(P=0.001$, 0.026, and 0.007, respectively). Multivariate analysis showed that ALK IHC-positivity was an adverse prognostic factor for disease-free survival (HR, 1.80; 95\% CI 1.182.77; $P=0.007)$, tumor-specific survival ( HR, 2.59; $95 \%$ CI $1.35-4.97 ; P=0.004)$, and overall survival (HR, 1.92; 95\% CI 1.07-3.44; $P=0.030)$.

Conclusion: The clinical characteristics of patients with ALK-positive lung adenocarcinoma were similar to those of EGFR-mutated patients. ALK rearrangement was an adverse prognostic factor in surgically-resected lung adenocarcinoma patients. 


\section{INTRODUCTION}

Lung cancer is the leading cause of cancer-related deaths in China [1]. Globally, non-small-cell lung cancer (NSCLC) accounts for $85 \%$ of lung cancer cases [2]. Although surgical resection may be curative in some patients, it is achievable only for early-stage NSCLC, and the majority of cases are diagnosed at an advanced stage. For advanced cases, cytotoxic chemotherapy is still the firstline treatment. In the last 5 years, evidence has accumulated that there are genetically-defined molecular subsets of lung adenocarcinoma, the most remarkable subset being patients with epidermal growth factor receptor $(E G F R)$ gene mutations, which defines a small subset of patients with NSCLC who have high sensitivity to EGFR-tyrosine kinase inhibitors (TKIs) such as gefitinib and erlotinib [3, 4].

Patients with echinoderm microtubule-associated protein-like 4 gene and anaplastic lymphoma kinase gene (EML4-ALK) fusion is another important subset of NSCLC. This is caused by a small inversion of chromosome $2 p$ (the $\mathrm{N}$-terminal half of EML4 encompassing the basic region) and the hydrophobic echinoderm microtubule-associated protein-like protein (HELP) domain, and a portion of the WD-repeat region becomes fused to the intracellular juxtamembrane region of $A L K$ [5]. The EML4- $A L K$ fusion gene possesses powerful oncogenic activity, both in vivo and in vitro [5,6]. It has been reported that there are 10 or more subtypes of the EML4-ALK fusion gene, with the E13:A20 and E6a/b:A20 types being the most common ones (incidence rates, 33\% and 29\%, respectively) [7, 8]. Recently, researchers have identified other $A L K$ fusion partners in addition to $E M L 4$, including TRK-fused gene (TFG) [9], kinesin family member 5B (KIF5B) [10], kinesin light chain $1(K L C l)$ [11], huntingtin-interacting protein 1 (HIPI) [12], translocated promoter region (TPR) [13], and SEC31A [14].

Methods of detecting $A L K$ gene fusion include immunohistochemistry (IHC), reverse transcriptasepolymerase chain reaction (RT-PCR) technology, and fluorescence in situ hybridization (FISH). In 2013, the National Comprehensive Cancer Network (NCCN) stated that FISH was the 'gold standard' method to detect $A L K$ fusion genes. However, FISH is expensive and it is difficult to determine the overall tumor morphology and heterogeneity with its use [15], while RT-PCR requires high-quality primers and more RNA [16]. In contrast, IHC is economical, practical, and efficient, and this method is now widely used in routine pathology laboratory testing. However, there is some subjectivity in evaluating staining results in IHC, and the accuracy of the method depends largely on the quality of the antibodies used [17]. Therefore, antibodies with high specificity and sensitivity are an important requirement. A recent study that compared 4 different $A L K$ antibodies - D5F3 (Ventana), D5F3 (CST), 1A4/1H7 (OriGene Tech), and 5A4 (Abcam) - reported that their sensitivities were $93.8 \%, 84.4 \%, 93.8 \%$, and $56.3 \%$, respectively [18]. Notably, the newly developed Ventana monoclonal antibody (D5F3) has greatly improved the specificity and sensitivity of IHC testing [19], and one study has suggested that it can be used as a stand-alone test in cases displaying an unequivocal staining pattern [20]. Recently, based on a fully automated IHC assay developed by Ventana Medical Systems, the Ventana ALK (D5F3) IHC kit was approved to detect $A L K$ fusion genes by the US Food and Drug Administration (FDA). The sensitivity and specificity of this IHC assay have been reported to be $100 \%$ and $98 \%$, respectively [21].

The prevalence of $A L K$ rearrangement in patients with NSCLC has been found to range from $1.4 \%$ to $13 \%$ [22-25], and to be most common in those with a young age, a never or light smoking history, an abundant signet ring cell or solid pattern histology, and wild-type $E G F R$ or $K R A S$ gene mutations [22-32]. The incidence rate of $A L K$ rearrangement in NSCLC with wild-type EGFR or KRAS gene mutations has been reported to range from $25.7 \%$ to $34 \%[22,29,33]$. Although crizotinib, a small-molecular TKI, is now approved for the treatment of advanced $A L K$-positive NSCLC in view of its favorable therapeutic effect and safety in clinical trials [34,35], the prognostic influence of $A L K$ rearrangement in early-stage NSCLC in the absence of crizotinib treatment remains unclear.

The aim of the present study was to detect overexpression of ALK protein with the Ventana IHC test and to examine the associations of $A L K$ rearrangement with clinicopathologic characteristics and treatment outcomes in patients with early-stage lung adenocarcinoma.

\section{RESULTS}

\section{Prevalence and clinicopathologic characteristics of patients harboring $A L K$ rearrangement}

Data on a total of 534 completely-resected lung adenocarcinoma patients were analyzed. The Ventana IHC test for $A L K$ rearrangement was performed in all patients. Fortytwo (7.9\%) of the 534 patients were IHC-positive for $A L K$.

The clinicopathologic characteristics of the 534 patients are summarized in Table 1. Two hundred and sixty-one patients $(48.8 \%)$ were male, and $273(51.2 \%)$ were female; 302 (56.6\%) who had smoked less than 100 cigarettes in their lifetime were classified as never-smokers, and $232(43.4 \%)$ were classified as were smokers. Tumor size $(\mathrm{cm})$ ranged from 0.2 to 12 . The pathologic stage was I in 309 patients (57.9\%), II in $53(9.9 \%)$, and III in $172(32.2 \%)$. The histopathologic subtypes, determined according to the new IASLC/ATS/ERS classification of lung adenocarcinoma [36], were adenocarcinoma in preinvasive lesions in 17 patients $(3.2 \%)$ and invasive adenocarcinoma in 481 patients (90.1\%), with lepidic predominant, acinar predominant, papillary predominant, micropapillary predominant, and solid predominant with mucin production subtypes present in 111 (20.8\%), 214 (40.1\%), 29 (5.4\%), 
$29(5.4 \%)$, and 98 patients (18.4\%), respectively, and variants of invasive adenocarcinoma in 36 patients $(6.7 \%)$. Postoperative adjuvant chemotherapy or radiotherapy was administered to 282 patients $(52.8 \%)$. ALK rearrangement was significantly associated with younger age (median age, 57.5 years in the $A L K$ IHC-positive group vs 60 years in the $A L K$ IHC-negative group; $P=0.011)$, high tumor status (pT4; $P=0.025$ ), high pathologic stage (IIIB; $P=0.002$ ), solid predominant adenocarcinoma with mucin production $(P=0.006)$, invasive mucinous adenocarcinoma $(P=$ $0.009)$, and receipt of adjuvant therapy after surgery $(P=$ 0.036). However, there were no significant associations with sex $(P=0.634)$, smoking status $(P=0.333)$, ECOG PS score $(P=0.587)$, tumor size $(P=0.955)$, and lymph node status $(P=0.131)$.

\section{Treatment outcomes}

The median follow-up duration for the patients studied was 29.0 months. At the time of analysis, 451 patients $(84.3 \%)$ were still alive, with $287(92.9 \%)$ in pathologic stage I, $41(77.4 \%)$ in pathologic stage II, and $123(71.5 \%)$ in pathologic stage III. However, 83 $(15.7 \%)$ of the 534 patients had died, $75(90.4 \%)$ of whom had tumor-related deaths, while $8(9.6 \%)$ had tumorunrelated deaths. Forty-one (49.4\%) of these patients were male and $42(50.6 \%)$ were female, while $47(56.6 \%)$ were never-smokers and $36(43.4 \%)$ were smokers. The pathologic stage was I in $22(26.5 \%)$ of the 83 patients, II in 12 patients $(14.5 \%)$, and III in 49 (59.0\%). The histopathologic subtypes were adenocarcinoma with atypical adenocarcinoma hyperplasia in 1 (1.2\%), lepidic predominant in $14(16.9 \%)$, acinar predominant in $30(36.1 \%)$, papillary predominant in $3(3.6 \%)$, micropapillary predominant in $4(4.8 \%)$, solid predominant with mucin production in $23(27.7 \%)$, invasive mucinous adenocarcinoma in $6(7.2 \%)$, and colloid in $2(2.5 \%)$. Fourteen patients $(16.9 \%)$ were $A L K$ IHC-positive, while $69(83.1 \%)$ were $A L K$ IHC-negative.

Mean overall survival was significantly shorter in $A L K$ IHC-positive patients than in the $A L K$ IHC-negative group (46.0 months vs 57.5 months, respectively; $P$ $=0.007)$. In addition, the overall mortality rate was significantly higher in the $A L K$ IHC-positive group than in the $A L K$ IHC-negative group $(33.3 \%$ vs $14.0 \%$, respectively; $P=0.003$ ), and the 2 -year overall survival rate after surgery was significantly lower (78.5\% vs $90.2 \%$, respectively; $P=0.032$ ). Moreover, mean tumorspecific survival was significantly shorter in the $A L K$ IHCpositive group than in the $A L K$ IHC-negative group (47.8 months vs 58.1 months, respectively; $P=0.026$ ), and the 2-year tumor-specific survival rate of $A L K$ IHC-positive patients was lower than that of $A L K$ IHC-negative patients (82.7\% vs $91.3 \%$, respectively; $P=0.092$ ).

Tumor recurrences occurred in a total of 199 patients $(37.3 \%), 25$ of whom $(12.6 \%)$ were in the $A L K$
IHC-positive group and $174(87.4 \%)$ in the $A L K$ IHCnegative group. The mean disease-free survival was 39.0 months in $A L K$ IHC-negative patients versus 26.3 months in $A L K$ IHC-positive patients $(P=0.001)$, and the tumor recurrence rate was significantly higher in the $A L K \mathrm{IHC}$ positive group $(59.5 \%$ vs $35.4 \%$, respectively; $P=0.003)$.

Clinical outcomes, including disease-free survival (DFS), tumor-specific survival (TSS), and overall survival (OS) in the 2 patient groups are shown in Figure 1, and the clinicopathologic characteristics of the 199 patients with recurrences are shown in Table 2. The median age of the $A L K$ IHC-positive group was significantly lower than that of the $A L K$ IHC-negative group (53 years vs 60 years, respectively; $P=0.002$ ). However, there were no significant differences for other clinicopathologic characteristics, such as sex $(P=0.391)$, smoking status $(P$ $=0.513)$, tumor status $(P=0.700)$, lymph node status $(P=$ $0.061)$, histopathologic subtypes $(P=0.070)$, or adjuvant therapy $(P=0.266)$. In addition, there were no statistically significant differences between the 2 groups for the first recurrence site $(P=0.865)$ and the number of recurrence sites $(P=0.328)$ [Table 2].

\section{Prognostic value of $A L K$ rearrangement in surgically-resected lung adenocarcinoma patients}

As shown in Table 3, univariate analysis indicated that disease-free, tumor-specific, and overall survival were significantly shorter in patients with a large tumor size, high tumor status, lymph node involvement, and solid predominant with mucin production adenocarcinoma. Adjuvant therapy was significantly associated with DFS (HR [hazard ratio], 1.93; 95\% CI 1.44-2.59; $P<0.001$ ) and TSS (HR, 0.17 ; 95\% CI 0.09-0.33; $P<0.001)$. ALK IHC-positivity was significantly associated with a shorter DFS (HR, 1.98; 95\% CI 1.30-3.01; $P=0.001)$, TSS (HR, $2.00 ; 95 \%$ CI 1.07-3.73; $P=0.029)$, and OS (HR, 2.16; $95 \%$ CI 1.21-3.85; $P=0.009)$.

Multivariate analysis using a Cox proportional hazards model compared the DFS, TSS, and OS of all patients. After adjusting for pathologic nodal staging, tumor staging, tumor size, and adenocarcinoma subtypes, the variables that remained significantly associated with a shorter DFS were high tumor status (HR, 1.55; 95\% CI $1.07-2.24 ; P=0.020)$ and lymph node involvement (HR, 2.53 ; 95\% CI 1.83-3.52; $P<0.001)$. A shorter tumorspecific survival was significantly associated with a large tumor size (HR, 1.17; 95\% CI 1.04-1.32; $P=0.012)$, high tumor status (HR, 2.49; 95\% CI 1.35-4.58; $P=0.003$ ), lymph node involvement (HR, 4.86; 95\% CI 2.93-8.04; $P<0.001$ ), solid predominant with mucin production adenocarcinoma (HR, 1.82; 95\% CI 1.07-3.07; $P=0.026)$, and no adjuvant therapy (HR, 0.06; 95\% CI 0.03-0.12; $P$ $<.001$ ). In addition, lymph node involvement (HR, 2.35; 95\% CI 1.48-3.75; $P<0.001)$ and solid predominant 
Table 1: Prevalence of $A L K$ rearrangement and its association with clinicopathologic characteristics in patients with early-stage lung adenocarcinoma

\begin{tabular}{|c|c|c|c|c|c|}
\hline & \multicolumn{4}{|c|}{ ALK D5F3 (Ventana) IHC } & \multirow[t]{3}{*}{$P$-value } \\
\hline & \multicolumn{2}{|l|}{ Positive } & \multicolumn{2}{|l|}{ Negative } & \\
\hline & No. of patients & $\%$ & No. of patients & $\%$ & \\
\hline Total & 42 & 7.9 & 492 & 92.1 & \\
\hline \multicolumn{6}{|l|}{ Sex: } \\
\hline Male & 19 & 45.2 & 242 & 49.2 & 0.634 \\
\hline Female & 23 & 54.8 & 250 & 50.8 & \\
\hline \multicolumn{6}{|l|}{ Age at surgery, years: } \\
\hline Median & 57.5 & & 60 & & $0.011^{\mathrm{a}}$ \\
\hline Range & $41-72$ & & $35-79$ & & \\
\hline \multicolumn{6}{|l|}{ Smoking status: } \\
\hline Never-smoker & 27 & 64.3 & 275 & 55.9 & 0.333 \\
\hline Smoker & 15 & 35.7 & 217 & 44.1 & \\
\hline \multicolumn{6}{|l|}{ ECOG PS: } \\
\hline 0 & 13 & 31.0 & 131 & 26.6 & 0.587 \\
\hline 1 & 29 & 69.0 & 361 & 73.4 & \\
\hline \multicolumn{6}{|l|}{$\begin{array}{l}\text { Family history of } \\
\text { cancer: }\end{array}$} \\
\hline No & 34 & 81.0 & 410 & 83.3 & 0.669 \\
\hline Yes & 8 & 19.0 & 82 & 16.7 & \\
\hline \multicolumn{6}{|l|}{$\begin{array}{l}\text { Localization of primary } \\
\text { tumor: }\end{array}$} \\
\hline Upper right lobe & 5 & 11.9 & 155 & 31.5 & 0.010 \\
\hline Middle right lobe & 5 & 11.9 & 47 & 9.6 & \\
\hline Lower right lobe & 13 & 31.0 & 96 & 19.5 & \\
\hline Upper left lobe & 6 & 14.3 & 109 & 22.2 & \\
\hline Lower left lobe & 10 & 23.8 & 72 & 14.6 & \\
\hline $\begin{array}{l}\text { Dragging in several } \\
\text { lobes }\end{array}$ & 3 & 7.1 & 13 & 2.6 & \\
\hline \multicolumn{6}{|l|}{ Operating methods: } \\
\hline \multicolumn{6}{|l|}{ Anatomy: } \\
\hline Wedge resection & 1 & 2.4 & 8 & 1.6 & 0.103 \\
\hline Sleeve resection & 1 & 2.4 & 4 & 0.8 & \\
\hline Segmentectomy & 0 & 0 & 7 & 1.4 & \\
\hline Lobectomy & 31 & 73.8 & 421 & 85.6 & \\
\hline Bilobectomy & 5 & 11.9 & 25 & 5.1 & \\
\hline Pneumonectomy & 2 & 4.8 & 8 & 1.6 & \\
\hline Others & 2 & 4.8 & 20 & 4.1 & \\
\hline
\end{tabular}

(Continued) 


\begin{tabular}{|c|c|c|c|c|c|}
\hline & \multicolumn{4}{|c|}{ ALK D5F3 (Ventana) IHC } & \multirow[t]{3}{*}{$P$-value } \\
\hline & \multicolumn{2}{|l|}{ Positive } & \multicolumn{2}{|l|}{ Negative } & \\
\hline & No. of patients & $\%$ & No. of patients & $\%$ & \\
\hline \multicolumn{6}{|l|}{ Technique: } \\
\hline Open thoracotomy & 29 & 69.0 & 350 & 71.1 & 0.860 \\
\hline Thoracoscopy & 13 & 31.0 & 142 & 28.9 & \\
\hline \multicolumn{6}{|l|}{ Tumor size, cm: } \\
\hline Median & 3 & & 3 & & $0.955^{\mathrm{a}}$ \\
\hline Range & $0.5-12$ & & $0.2-12$ & & \\
\hline \multicolumn{6}{|l|}{ Tumor status: } \\
\hline $\mathrm{T} 1$ & 21 & 50.0 & 295 & 60.0 & 0.102 \\
\hline $\mathrm{T} 2$ & 13 & 31.0 & 139 & 28.3 & \\
\hline $\mathrm{T} 3$ & 1 & 2.4 & 26 & 5.3 & \\
\hline $\mathrm{T} 4$ & 7 & 16.7 & 32 & 6.5 & \\
\hline \multicolumn{6}{|l|}{ Lymph node status: } \\
\hline N0 & 22 & 52.4 & 320 & 65.0 & 0.034 \\
\hline N1 & 2 & 4.8 & 37 & 7.5 & \\
\hline N2 & 16 & 38.1 & 132 & 26.8 & \\
\hline $\mathrm{N} 3$ & 2 & 4.8 & 3 & 0.6 & \\
\hline \multicolumn{6}{|l|}{ Stage: } \\
\hline IA & 11 & 26.2 & 226 & 45.9 & 0.008 \\
\hline IB & 7 & 16.7 & 65 & 13.2 & \\
\hline IIA & 5 & 11.9 & 40 & 8.1 & \\
\hline IIB & 0 & 0 & 8 & 1.6 & \\
\hline IIIA & 11 & 26.2 & 129 & 26.2 & \\
\hline IIIB & 8 & 19.0 & 24 & 4.9 & \\
\hline \multicolumn{6}{|l|}{$\begin{array}{l}\text { Histopathologic } \\
\text { subtypes: }\end{array}$} \\
\hline Preinvasive lesions: & & & & & 0.006 \\
\hline $\begin{array}{l}\text { Atypical } \\
\text { adenocarcinoma } \\
\text { hyperplasia }\end{array}$ & 0 & 0 & 1 & 0.2 & \\
\hline $\begin{array}{l}\text { Adenocarcinoma } \\
\text { in situ }\end{array}$ & 1 & 2.4 & 5 & 1.0 & \\
\hline $\begin{array}{l}\text { Minimally } \\
\text { invasive } \\
\text { adenocarcinoma }\end{array}$ & 0 & 0 & 10 & 2.0 & \\
\hline \multicolumn{6}{|l|}{$\begin{array}{l}\text { Invasive } \\
\text { adenocarcinoma: }\end{array}$} \\
\hline $\begin{array}{l}\text { Lepidic } \\
\text { predominant }\end{array}$ & 4 & 9.5 & 107 & 21.7 & \\
\hline
\end{tabular}

(Continued) 
ALK D5F3 (Ventana) IHC

$P$-value

\begin{tabular}{|c|c|c|c|c|c|}
\hline & \multicolumn{4}{|c|}{ ALK D5F3 (Ventana) IHC } & \multirow[t]{3}{*}{$P$-value } \\
\hline & \multicolumn{2}{|l|}{ Positive } & \multicolumn{2}{|l|}{ Negative } & \\
\hline & No. of patients & $\%$ & No. of patients & $\%$ & \\
\hline $\begin{array}{c}\text { Acinar } \\
\text { predominant }\end{array}$ & 9 & 21.4 & 205 & 41.7 & \\
\hline $\begin{array}{l}\text { Papillary } \\
\text { predominant }\end{array}$ & 3 & 7.1 & 26 & 5.3 & \\
\hline $\begin{array}{l}\text { Micropapillary } \\
\text { predominant }\end{array}$ & 3 & 7.1 & 26 & 5.3 & \\
\hline $\begin{array}{l}\text { Solid predominant } \\
\text { with mucin production }\end{array}$ & 15 & 35.7 & 83 & 16.9 & $0.006^{\mathrm{b}}$ \\
\hline \multicolumn{6}{|l|}{$\begin{array}{l}\text { Variants of invasive } \\
\text { adenocarcinoma: }\end{array}$} \\
\hline $\begin{array}{l}\text { Invasive mucinous } \\
\text { adenocarcinoma }\end{array}$ & 7 & 16.7 & 25 & 5.1 & $0.009^{c}$ \\
\hline Colloid & 0 & 0 & 2 & 0.4 & \\
\hline Enteric & 0 & 0 & 2 & 0.4 & \\
\hline \multicolumn{6}{|l|}{ Adjuvant therapy: } \\
\hline No & 13 & 31.0 & 239 & 48.6 & 0.036 \\
\hline Yes & 29 & 69.0 & 253 & 51.4 & \\
\hline
\end{tabular}

${ }^{a}$ Wilcoxon rank-sum test.

${ }^{\mathrm{b}}$ Comparison between solid predominant with mucin production and other types.

${ }^{\mathrm{c}}$ Comparison between invasive mucinous adenocarcinoma and other types.

$A L K$, anaplastic lymphoma kinase; ECOG PS, Eastern Cooperative Oncology Group performance status; IHC, immunohistochemistry.

with mucin production adenocarcinoma (HR, 1.77; $95 \%$ CI 1.08-2.90; $P=0.023$ ) were adverse prognostic factors for OS. Multivariate analysis showed that ALK IHCpositivity was significantly associated with a shorter DFS (HR, 1.80; 95\% CI 1.18-2.77; $P=0.007$ ), TSS (HR, 2.59; 95\% CI 1.35-4.97; $P=0.004)$, and OS (HR, $1.92 ; 95 \%$ CI 1.07-3.44; $P=0.030$ ). These results indicate that $A L K$ rearrangement is an independent adverse prognostic factor in surgically-resected lung adenocarcinoma patients.

We further analyzed factors associated with recurrences in $A L K$ IHC-positive and ALK IHC-negative patients. As shown in Table 4, univariate analysis indicated that lymph node involvement (HR, 3.56; 95\% CI 1.51-8.38; $P=0.004$ ) was an adverse prognostic factor for tumor recurrences in the $A L K$ IHC-positive group, and a large tumor size (HR, 1.17; 95\% CI 1.08-1.27; $P<$ 0.001 ), high tumor status (HR, 2.33; 95\% CI 1.60-3.40; $P<0.001$ ), lymph node involvement (HR, 2.84; $95 \%$ CI 2.10-3.83; $P<0.001$ ), solid predominant with mucin production adenocarcinoma (HR, 1.63; 95\% CI 1.132.34; $P=0.008$ ), and adjuvant therapy (HR, $1.89 ; 95 \%$ CI 1.39-2.57; $P<0.001$ ) were adverse prognostic factors for tumor recurrences in the $A L K$ IHC-negative group.
With multivariate analysis, lymph node involvement was found to be an independent adverse prognostic factor for disease-free survival in ALK IHC-negative patients (HR, 2.32; 95\% CI 1.64-3.29; $P<0.001)$.

\section{DISCUSSION}

Previous studies have shown that the prevalence of $A L K$ rearrangement in early-stage NSCLC ranges from $2.4 \%$ to $8.6 \%$, depending on the detection method used and the population studied [28-32]. We found that the prevalence was $7.9 \%$, which is consistent with other studies. $A L K$ rearrangement has previously been shown to be more common in light smokers or never-smokers [22-30], females [23-25, 28], the solid predominant with mucin production adenocarcinoma subtype [37-39], and young patients $[22,24,26,28,30,31]$. However, $A L K$ rearrangement has been reported in males $(P=0.039)$ [22] and some studies have observed no sex difference [23, 26, 29-31]. Furthermore, one study concluded that $A L K$ rearrangement is not related to smoking status [31]. A recent meta-analysis [40] showed that the prevalence of $A L K$ rearrangement in stage III-IV NSCLC is higher than that 
Table 2: Clinicopathologic characteristics of 199 patients with recurrent lung adenocarcinoma

\begin{tabular}{|c|c|c|c|c|c|}
\hline & \multicolumn{4}{|c|}{ ALK D5F3 (Ventana) IHC } & \multirow[t]{3}{*}{$P$-value } \\
\hline & \multicolumn{2}{|l|}{ Positive } & \multicolumn{2}{|l|}{ Negative } & \\
\hline & No. of patients & $\%$ & No. of patients & $\%$ & \\
\hline Tumor recurrence & 25 & 59.5 & 174 & 35.4 & 0.003 \\
\hline \multicolumn{6}{|l|}{ Sex: } \\
\hline Male & 9 & 36.0 & 82 & 47.1 & 0.391 \\
\hline Female & 16 & 64.0 & 92 & 52.9 & \\
\hline \multicolumn{6}{|l|}{ Age at surgery, years: } \\
\hline Median & 53 & & 60 & & $0.002^{\mathrm{a}}$ \\
\hline Range & $41-71$ & & $35-77$ & & \\
\hline \multicolumn{6}{|l|}{ Smoking status: } \\
\hline Never-smoker & 17 & 68.0 & 102 & 58.6 & 0.513 \\
\hline Smoker & 8 & 32.0 & 72 & 41.4 & \\
\hline \multicolumn{6}{|l|}{ ECOG PS: } \\
\hline 0 & 9 & 36.0 & 44 & 25.3 & 0.332 \\
\hline 1 & 16 & 64.0 & 130 & 74.7 & \\
\hline \multicolumn{6}{|l|}{ Family history of cancer: } \\
\hline No & 18 & 72.0 & 144 & 82.8 & 0.268 \\
\hline Yes & 7 & 28.0 & 30 & 17.2 & \\
\hline \multicolumn{6}{|l|}{ Localization of primary tumor: } \\
\hline Upper right lobe & 4 & 16.0 & 47 & 27.0 & 0.171 \\
\hline Middle right lobe & 1 & 4.0 & 16 & 9.2 & \\
\hline Lower right lobe & 8 & 32.0 & 39 & 22.4 & \\
\hline Upper left lobe & 3 & 12.0 & 39 & 22.4 & \\
\hline Lower left lobe & 8 & 32.0 & 24 & 13.8 & \\
\hline Dragging in several lobes & 1 & 4.0 & 9 & 5.2 & \\
\hline \multicolumn{6}{|l|}{ Operating methods: } \\
\hline \multicolumn{6}{|l|}{ Anatomy: } \\
\hline Wedge resection & 0 & 0.0 & 2 & 1.1 & 0.769 \\
\hline Sleeve resection & 0 & 0.0 & 3 & 1.7 & \\
\hline Segmentectomy & 0 & 0.0 & 1 & 0.6 & \\
\hline Lobectomy & 21 & 84.0 & 136 & 78.2 & \\
\hline Bilobectomy & 1 & 4.0 & 14 & 8.0 & \\
\hline Pneumonectomy & 2 & 8.0 & 5 & 2.9 & \\
\hline Others & 1 & 4.0 & 13 & 7.5 & \\
\hline \multicolumn{6}{|l|}{ Technique: } \\
\hline Open thoracotomy & 17 & 68.0 & 126 & 72.4 & 0.640 \\
\hline Thoracoscopy & 8 & 32.0 & 48 & 27.6 & \\
\hline
\end{tabular}

(Continued) 


\begin{tabular}{|c|c|c|c|c|c|}
\hline & \multicolumn{4}{|c|}{ ALK D5F3 (Ventana) IHC } & \multirow[t]{3}{*}{$P$-value } \\
\hline & \multicolumn{2}{|l|}{ Positive } & \multicolumn{2}{|l|}{ Negative } & \\
\hline & No. of patients & $\%$ & No. of patients & $\%$ & \\
\hline \multicolumn{6}{|l|}{ Tumor size, cm: } \\
\hline Median & 3 & & 3 & & $0.690^{\mathrm{a}}$ \\
\hline Range & $0.7-12$ & & $0.3-12$ & & \\
\hline \multicolumn{6}{|l|}{ Tumor status: } \\
\hline $\mathrm{T} 1$ & 12 & 48.0 & 83 & 47.7 & 0.700 \\
\hline $\mathrm{T} 2$ & 7 & 28.0 & 57 & 32.8 & \\
\hline $\mathrm{T} 3$ & 1 & 4.0 & 13 & 7.5 & \\
\hline $\mathrm{T} 4$ & 5 & 20.0 & 21 & 12.1 & \\
\hline \multicolumn{6}{|l|}{ Lymph node status: } \\
\hline No & 8 & 32.0 & 77 & 44.3 & 0.061 \\
\hline N1 & 1 & 4.0 & 14 & 8.0 & \\
\hline $\mathrm{N} 2$ & 14 & 56.0 & 82 & 47.1 & \\
\hline N3 & 2 & 8.0 & 1 & 0.6 & \\
\hline \multicolumn{6}{|l|}{ Stage: } \\
\hline IA & 4 & 16.0 & 51 & 29.3 & 0.118 \\
\hline IB & 3 & 12.0 & 11 & 6.3 & \\
\hline IIA & 2 & 8.0 & 17 & 9.8 & \\
\hline IIB & 0 & 0.0 & 4 & 2.3 & \\
\hline IIIA & 9 & 36.0 & 74 & 42.5 & \\
\hline IIIB & 7 & 28.0 & 17 & 9.8 & \\
\hline \multicolumn{6}{|l|}{ Histopathologic subtypes: } \\
\hline Preinvasive lesions: & & & & & 0.070 \\
\hline Adenocarcinoma in situ & 1 & 4.0 & 3 & 1.7 & \\
\hline $\begin{array}{l}\text { Minimally invasive } \\
\text { adenocarcinoma }\end{array}$ & 0 & 0.0 & 1 & 0.6 & \\
\hline \multicolumn{6}{|l|}{ Invasive adenocarcinoma: } \\
\hline Lepidic predominant & 2 & 8.0 & 29 & 16.7 & \\
\hline Acinar predominant & 5 & 20.0 & 70 & 40.2 & \\
\hline Papillary predominant & 1 & 4.0 & 6 & 3.4 & \\
\hline $\begin{array}{l}\text { Micropapillary } \\
\text { predominant }\end{array}$ & 3 & 12.0 & 17 & 9.8 & \\
\hline $\begin{array}{l}\text { Solid predominant with } \\
\text { mucin production }\end{array}$ & 8 & 32.0 & 38 & 21.8 & \\
\hline \multicolumn{6}{|l|}{$\begin{array}{l}\text { Variants of invasive } \\
\text { adenocarcinoma: }\end{array}$} \\
\hline $\begin{array}{l}\text { Invasive mucinous } \\
\text { adenocarcinoma }\end{array}$ & 5 & 20.0 & 7 & 4.0 & \\
\hline
\end{tabular}




\begin{tabular}{|c|c|c|c|c|c|}
\hline & \multicolumn{4}{|c|}{ ALK D5F3 (Ventana) IHC } & \multirow[t]{3}{*}{$P$-value } \\
\hline & \multicolumn{2}{|l|}{ Positive } & \multicolumn{2}{|l|}{ Negative } & \\
\hline & No. of patients & $\%$ & No. of patients & $\%$ & \\
\hline Colloid & 0 & 0.0 & 2 & 1.1 & \\
\hline Enteric & 0 & 0.0 & 1 & 0.6 & \\
\hline \multicolumn{6}{|l|}{ Adjuvant therapy: } \\
\hline No & 6 & 24.0 & 64 & 36.8 & 0.266 \\
\hline Yes & 19 & 76.0 & 110 & 63.2 & \\
\hline \multicolumn{6}{|c|}{ First site of recurrence: } \\
\hline Lung & 9 & 36.0 & 58 & 33.3 & 0.865 \\
\hline Bone & 4 & 16.0 & 33 & 19.0 & \\
\hline Brain & 5 & 20.0 & 29 & 16.7 & \\
\hline Pleura & 1 & 4.0 & 17 & 9.8 & \\
\hline Liver & 1 & 4.0 & 14 & 8.0 & \\
\hline Others & 5 & 20.0 & 23 & 13.2 & \\
\hline \multicolumn{6}{|c|}{ Number of recurrence sites: } \\
\hline Single site & 20 & 80.0 & 154 & 88.5 & 0.328 \\
\hline Multiple sites & 5 & 20.0 & 20 & 11.5 & \\
\hline
\end{tabular}

${ }^{a}$ Wilcoxon rank-sum test.

$A L K$, anaplastic lymphoma kinase; ECOG PS, Eastern Cooperative Oncology Group performance status; IHC, immunohistochemistry.

in stages I-II, indicating that $A L K$ rearrangement is more common with higher pathologic stages, which is consistent with our results (IIIB, $P=0.002$ ). Although a previous study [28] showed that $A L K$-positive lung cancers have unique biologic features with early nodal metastasis despite a small-sized primary tumor (pT1, $P=0.02$ ), we found that $A L K$ IHC-positive lung adenocarcinoma is associated with a higher tumor stage (pT4, $P=0.025)$. As factors such as race, population, detection method, and the subsequent statistical analysis were not the same in the above studies, this may be the cause of the reported differences. Further larger-scale studies are necessary to resolve these differences.

The univariate analysis for disease-free survival in our study showed that patients receiving adjuvant therapy had a higher risk of disease recurrence $(P<$ $0.001)$. However, this does not mean that they would not benefit from adjuvant therapy. Among the 199 patients with recurrences in our study population, 129 (64.8\%) had received adjuvant therapy. Of these 129 patients, $25(19.4 \%)$ were in pathologic stage I, and $104(80.6 \%)$ were in pathologic stages II and III. As the use of adjuvant therapy is stage-dependent, patients with a high pathological stage comprised a high proportion $(80.6 \%)$ of all patients who received adjuvant therapy. These patients are known to have a poor prognosis.
There is controversy as to whether $A L K$ rearrangement is associated with the prognosis of patients with early-stage NSCLC. A retrospective study from South Korea [29] reported that $A L K$ rearrangement was not associated with overall survival in lung adenocarcinoma patients who were never-smokers $(P$ $=0.720)$, but disease-free survival was shorter in $A L K$ positive patients $(P=0.022)$. Another study, also from South Korea [28], concluded that $A L K$ rearrangement was not a prognostic factor in early-stage NSCLC patients, and a study conducted in Japan [32] came to the same conclusion. The populations of these studies were all Asian. In comparison, a study from European Thoracic Oncology Platform Lungscape Project [30] found that $A L K$-positivity was related to an improved overall survival in early-stage lung adenocarcinoma patients. However, we found that $A L K$ rearrangement was associated with worse disease-free survival, tumor-specific survival, and overall survival in surgically-resected lung adenocarcinoma patients. Many studies have shown that the prognosis of patients with solid predominant with mucin production adenocarcinoma is poor [41-46]. In our study, 15 (35.7\%) of the $42 A L K$ IHC-positive patients had solid predominant with mucin production adenocarcinoma, and analysis of clinicopathologic characteristics showed that 
A

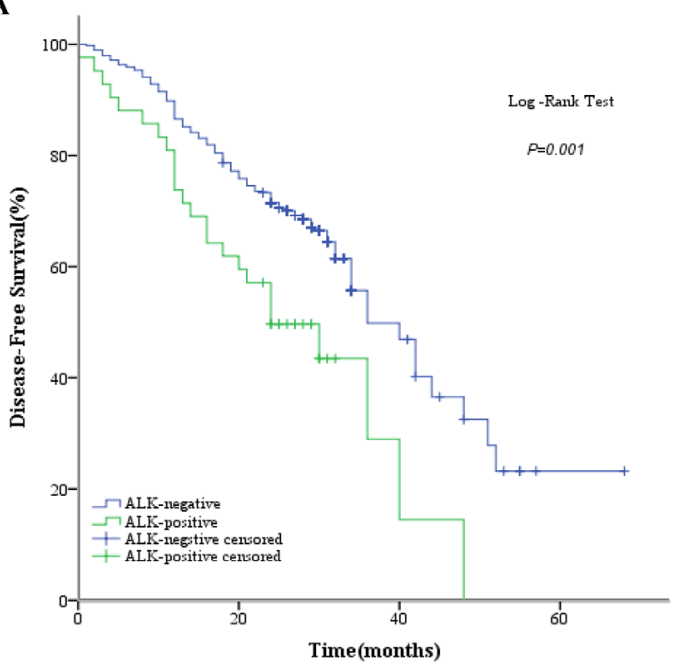

B


Figure 1: Kaplan-Meier curves showing (A) disease-free survival (DFS) by ALK D5F3 (Ventana) IHC; (B) tumor-specific survival (TSS) by ALK D5F3 (Ventana) IHC; and (C) overall survival (OS) by ALK D5F3 (Ventana) IHC. 
Table 3: Disease-free, tumor-specific, and overall survival analyses for the 534 surgically-resected lung adenocarcinoma patients

\begin{tabular}{|c|c|c|c|c|c|c|c|c|c|c|c|c|c|c|c|}
\hline & \multirow{2}{*}{$\begin{array}{c}\text { No. of } \\
\text { recurrences }\end{array}$} & \multicolumn{4}{|c|}{ HR for recurrence $(95 \% \mathrm{CI})$} & \multirow{2}{*}{$\begin{array}{l}\text { No. of tumor } \\
\text { related deaths }\end{array}$} & \multicolumn{4}{|c|}{ HR for tumor-related death $(95 \% \mathrm{CI})$} & \multirow[t]{2}{*}{ No. of deaths } & \multicolumn{4}{|c|}{ HR for death $(95 \% \mathrm{CI})$} \\
\hline & & Univariate & $P$-value & Multivariate & $P$-value & & Univariate & $P$-value & Multivariate & $P$-value & & Univariate & $P$-value & Multivariate & $P$-value \\
\hline \multicolumn{16}{|l|}{ Sex: } \\
\hline Male & 91 & 1000 (ref) & & & & 36 & 1000 (ref) & & & & 41 & 1000 (ref) & & & \\
\hline Female & 108 & $\begin{array}{c}1.07 \\
(0.81-1.41)\end{array}$ & 0.650 & & & 39 & $\begin{array}{c}0.92(0.58- \\
1.45)\end{array}$ & 0.720 & & & 42 & $\begin{array}{c}0.88(0.57- \\
1.36)\end{array}$ & 0.561 & & \\
\hline \multirow[t]{2}{*}{$\begin{array}{l}\text { Age at surgery, } \\
\text { years }\end{array}$} & & 1000 (ref) & & & & & 1000 (ref) & & & & & 1000 (ref) & & & \\
\hline & 199 & $\begin{array}{c}0.85 \\
(0.64-1.12)\end{array}$ & 0.237 & & & 75 & $\begin{array}{c}0.99(0.97- \\
1.02)\end{array}$ & 0.650 & & & 83 & $\begin{array}{c}1.11(0.72- \\
1.71)\end{array}$ & 0.652 & & \\
\hline \multicolumn{16}{|l|}{ Smoking status: } \\
\hline Never smoker & 119 & 1000 (ref) & & & & 45 & 1000 (ref) & & & & 47 & 1000 (ref) & & & \\
\hline Smoker & 80 & $\begin{array}{c}0.93 \\
(0.70-1.24)\end{array}$ & 0.632 & & & 30 & $\begin{array}{c}1.03(0.64- \\
1.63)\end{array}$ & 0.917 & & & 36 & $\begin{array}{c}1.16(0.75- \\
1.80)\end{array}$ & 0.496 & & \\
\hline \multicolumn{16}{|l|}{ ECOG PS: } \\
\hline 0 & 53 & $1000(\mathrm{ref})$ & & & & 22 & 1000 (ref) & & & & 24 & 1000 (ref) & & & \\
\hline 1 & 146 & $\begin{array}{c}1.09 \\
(0.79-1.49)\end{array}$ & 0.599 & & & 53 & $\begin{array}{c}0.94(0.57- \\
1.54)\end{array}$ & 0.797 & & & 59 & $\begin{array}{c}0.95(0.59- \\
1.53)\end{array}$ & 0.837 & & \\
\hline \multicolumn{16}{|l|}{ Technique: } \\
\hline $\begin{array}{l}\text { Open } \\
\text { thoracotomy }\end{array}$ & 143 & 1000 (ref) & & & & 56 & 1000 (ref) & & & & 62 & 1000 (ref) & & & \\
\hline Thorascopy & 56 & $\begin{array}{c}0.85 \\
(0.62-1.16)\end{array}$ & 0.293 & & & 19 & $\begin{array}{c}0.73(0.43- \\
1.23)\end{array}$ & 0.235 & & & 21 & $\begin{array}{c}0.74(0.45- \\
1.21)\end{array}$ & 0.224 & & \\
\hline \multirow[t]{2}{*}{ Tumor size, cm: } & & 1000 (ref) & & & & & 1000 (ref) & & & & & $1000(\mathrm{ref})$ & & & \\
\hline & 199 & $\begin{array}{c}1.41 \\
(1.07-1.88)\end{array}$ & 0.016 & $\begin{array}{c}1.18(0.88- \\
1.59)\end{array}$ & 0.266 & 75 & $\begin{array}{c}1.23(1.11- \\
1.36)\end{array}$ & $<0.001$ & $\begin{array}{c}1.17(1.04- \\
1.32)\end{array}$ & 0.012 & 83 & $\begin{array}{l}1.79(1.14- \\
2.81)\end{array}$ & 0.012 & $\begin{array}{c}1.48(0.94- \\
2.35)\end{array}$ & 0.094 \\
\hline \multicolumn{16}{|l|}{ Tumor status: } \\
\hline $\mathrm{T} 1-2$ & 159 & 1000 (ref) & & & & 57 & 1000 (ref) & & & & 64 & 1000 (ref) & & & \\
\hline T3-4 & 40 & $\begin{array}{c}2.39 \\
(1.69-3.39)\end{array}$ & $<0.001$ & $\begin{array}{l}1.55(1.07- \\
2.24)\end{array}$ & 0.020 & 18 & $\begin{array}{c}2.67(1.57- \\
4.54)\end{array}$ & $<0.001$ & $\begin{array}{c}2.49(1.35- \\
4.58)\end{array}$ & 0.003 & 19 & $\begin{array}{c}2.47(1.48- \\
4.14)\end{array}$ & 0.001 & $\begin{array}{l}1.62(0.94- \\
2.79)\end{array}$ & 0.082 \\
\hline \multicolumn{16}{|l|}{$\begin{array}{l}\text { Lymph node } \\
\text { status: }\end{array}$} \\
\hline Negative & 85 & 1000 (ref) & & & & 30 & 1000 (ref) & & & & 33 & 1000 (ref) & & & \\
\hline Positive & 114 & $\begin{array}{c}3.00 \\
(2.26-3.98)\end{array}$ & $<0.001$ & $\begin{array}{l}2.53(1.83- \\
3.52)\end{array}$ & $<0.001$ & 45 & $\begin{array}{l}2.78(1.75- \\
4.42)\end{array}$ & $<0.001$ & $\begin{array}{l}4.86(2.93- \\
8.04)\end{array}$ & $<0.001$ & 50 & $\begin{array}{c}2.82(1.81- \\
4.38)\end{array}$ & $<0.001$ & $\begin{array}{l}2.35(1.48- \\
3.75)\end{array}$ & $<0.001$ \\
\hline \multicolumn{16}{|l|}{$\begin{array}{l}\text { Adenocarcinoma } \\
\text { subtypes: }\end{array}$} \\
\hline Others & 153 & 1000 (ref) & & & & 54 & 1000 (ref) & & & & 60 & 1000 (ref) & & & \\
\hline $\begin{array}{l}\text { Solid } \\
\text { predominant with } \\
\text { mucin production }\end{array}$ & 46 & $\begin{array}{c}1.60 \\
(1.15-2.23)\end{array}$ & 0.006 & $\begin{array}{c}1.35(0.97- \\
1.89)\end{array}$ & 0.080 & 21 & $\begin{array}{l}2.12(1.27- \\
3.53)\end{array}$ & 0.004 & $\begin{array}{c}1.82(1.07- \\
3.07)\end{array}$ & 0.026 & 23 & $\begin{array}{c}2.06(1.27- \\
3.34)\end{array}$ & 0.004 & $\begin{array}{c}1.77(1.08- \\
2.90)\end{array}$ & 0.023 \\
\hline \multicolumn{16}{|l|}{ Adjuvant therapy: } \\
\hline No & 70 & $1000(\mathrm{ref})$ & & & & 65 & 1000 (ref) & & & & 31 & $1000(\mathrm{ref})$ & & & \\
\hline Yes & 129 & $\begin{array}{c}1.93 \\
(1.44-2.59)\end{array}$ & $<0.001$ & $\begin{array}{c}1.08(0.76- \\
1.52)\end{array}$ & 0.679 & 10 & $\begin{array}{c}0.17(0.09- \\
0.33)\end{array}$ & $<0.001$ & $\begin{array}{c}0.06(0.03- \\
0.12)\end{array}$ & $<0.001$ & 52 & $\begin{array}{c}1.50(0.96- \\
2.33)\end{array}$ & 0.077 & & \\
\hline \multicolumn{16}{|l|}{$\begin{array}{l}A L K \\
\text { rearrangement: }\end{array}$} \\
\hline Negative & 174 & 1000 (ref) & & & & 63 & 1000 (ref) & & & & 69 & 1000 (ref) & & & \\
\hline Positive & 25 & $\begin{array}{c}1.98 \\
(1.30-3.01)\end{array}$ & 0.001 & $\begin{array}{l}1.80(1.18- \\
2.77)\end{array}$ & 0.007 & 12 & $\begin{array}{c}2.00(1.07- \\
3.73)\end{array}$ & 0.029 & $\begin{array}{c}2.59(1.35- \\
4.97)\end{array}$ & 0.004 & 14 & $\begin{array}{c}2.16(1.21- \\
3.85)\end{array}$ & 0.009 & $\begin{array}{c}1.92(1.07- \\
3.44)\end{array}$ & 0.030 \\
\hline
\end{tabular}

$A L K$, anaplastic lymphoma kinase; $C I$, confidence interval; ECOG PS, Eastern Cooperative Oncology Group performance status; $H R$, hazard ratio.

$A L K$ rearrangement was significantly more common in patients with solid predominant with mucin production adenocarcinoma than in patients with other subtypes $(P=$ 0.006). In addition, univariate analysis showed that solid predominant with mucin production adenocarcinoma was associated with a shorter DFS, TSS, and OS, and multivariate analysis showed that this subtype was an adverse prognostic factors for TSS (HR, 1.82; 95\% 
Table 4: Disease-free survival analysis for the $A L K$ IHC-positive and $A L K$ IHC-negative resected lung adenocarcinoma patients

\begin{tabular}{|c|c|c|c|c|c|c|c|c|}
\hline & \multicolumn{3}{|c|}{ ALK IHC-positive } & \multicolumn{5}{|c|}{$A L K$ IHC-negative } \\
\hline & \multirow[t]{2}{*}{$\begin{array}{l}\text { No. of } \\
\text { recurrences }\end{array}$} & \multicolumn{2}{|c|}{$\begin{array}{l}\text { HR for recurrence } \\
(95 \% \mathrm{CI})\end{array}$} & \multirow[t]{2}{*}{$\begin{array}{c}\text { No. of } \\
\text { recurrences }\end{array}$} & \multicolumn{4}{|c|}{ HR for recurrence $(95 \% \mathrm{CI})$} \\
\hline & & Univariate & $P$-value & & Univariate & $P$-value & Multivariate & $P$-value \\
\hline \multicolumn{9}{|l|}{ Sex: } \\
\hline Male & 9 & 1000 (ref) & & 82 & 1000 (ref) & & & \\
\hline Female & 16 & $1.66(0.71-3.89)$ & 0.241 & 92 & $0.99(0.73-1.33)$ & 0.944 & & \\
\hline \multirow[t]{2}{*}{ Age at surgery, years } & & 1000 (ref) & & & 1000 (ref) & & & \\
\hline & 25 & $1.01(0.95-1.09)$ & 0.688 & 174 & $1.00(0.98-1.02)$ & 0.769 & & \\
\hline \multicolumn{9}{|l|}{ Smoking status: } \\
\hline Never smoker & 17 & 1000 (ref) & & 102 & 1000 (ref) & & & \\
\hline Smoker & 8 & $0.77(0.32-1.87)$ & 0.563 & 72 & $0.99(0.73-1.34)$ & 0.944 & & \\
\hline \multicolumn{9}{|l|}{ ECOG PS: } \\
\hline 0 & 9 & 1000 (ref) & & 44 & 1000 (ref) & & & \\
\hline 1 & 16 & $1.18(0.48-2.91)$ & 0.714 & 130 & $1.16(0.82-1.63)$ & 0.401 & & \\
\hline \multicolumn{9}{|l|}{ Technique: } \\
\hline Open thoracotomy & 29 & 1000 (ref) & & 126 & 1000 (ref) & & & \\
\hline Thorascopy & 13 & $0.85(0.36-2.00)$ & 0.708 & 48 & $0.83(0.59-1.16)$ & 0.269 & & \\
\hline \multirow[t]{2}{*}{ Tumor size, cm: } & & 1000 (ref) & & & 1000 (ref) & & 1000 (ref) & \\
\hline & 25 & $1.06(0.86-1.30)$ & 0.619 & 174 & $1.17(1.08-1.27)$ & $<0.001$ & $1.05(0.96-1.14)$ & 0.316 \\
\hline \multicolumn{9}{|l|}{ Tumor status: } \\
\hline $\mathrm{T} 1-2$ & 19 & 1000 (ref) & & 140 & 1000 (ref) & & 1000 (ref) & \\
\hline $\mathrm{T} 3-4$ & 6 & $2.60(0.99-6.81)$ & 0.051 & 34 & $2.33(1.60-3.40)$ & $<0.001$ & $1.47(0.96-2.27)$ & 0.079 \\
\hline \multicolumn{9}{|l|}{ Lymph node status: } \\
\hline Negative & 8 & 1000 (ref) & & 77 & 1000 (ref) & & 1000 (ref) & \\
\hline Positive & 17 & $3.56(1.51-8.38)$ & 0.004 & 97 & $2.84(2.10-3.83)$ & $<0.001$ & $2.32(1.64-3.29)$ & $<0.001$ \\
\hline \multicolumn{9}{|l|}{ Adenocarcinoma subtypes: } \\
\hline Others & 17 & 1000 (ref) & & 136 & 1000 (ref) & & 1000 (ref) & \\
\hline $\begin{array}{l}\text { Solid predominant with } \\
\text { mucin production }\end{array}$ & 8 & $1.15(0.48-2.74)$ & 0.758 & 38 & $1.63(1.13-2.34)$ & 0.008 & $1.38(0.95-2.01)$ & 0.089 \\
\hline \multicolumn{9}{|l|}{ Adjuvant therapy: } \\
\hline No & 6 & 1000 (ref) & & 64 & 1000 (ref) & & 1000 (ref) & \\
\hline Yes & 19 & $1.54(0.61-3.92)$ & 0.365 & 110 & $1.89(1.39-2.57)$ & $<0.001$ & $1.13(0.79-1.62)$ & 0.494 \\
\hline
\end{tabular}

$A L K$, anaplastic lymphoma kinase; $C I$, confidence interval; ECOG PS, Eastern Cooperative Oncology Group performance status; HR, hazard ratio.

CI $1.07-3.07 ; P=0.026)$ and $\mathrm{OS}(\mathrm{HR}, 1.77 ; 95 \% \mathrm{CI}$ $1.08-2.90 ; P=0.020)$. Therefore, whether the poor prognosis of $A L K$-positive patients is associated with the histopathologic subtype is uncertain.

To our knowledge, this is the largest cohort of patients in which $A L K$ rearrangements have been evaluated by a fully-automated immunochemistry assay in patients with early-stage lung adenocarcinoma, unlike most other $A L K$-related studies focusing on late-stage patients. We concluded that $A L K$-positivity is correlated with worse disease-free, tumor-specific, and overall survival. In addition, this is the first study to conduct a detailed clinicopathologic analysis of Chinese patients, and its findings complement existing information on the influence of $A L K$ rearrangement on the prognosis of patients with early-stage NSCLC. However, like most retrospective studies of rare genes, our study has some limitations including observational assessment of tumor 
recurrence, unknown $E G F R$ or $K R A S$ status, and a short follow-up time, Therefore, in a future study, we will obtain treatment information after tumor recurrences, analyze other rare genes, and also analyze the relationship of the solid predominant with mucin production and invasive mucinous adenocarcinoma subtypes with $A L K$ rearrangement and treatment outcome. In addition, the follow-up duration will be extended to obtain more mature data for the overall survival analysis.

In summary, the prevalence of $A L K$ rearrangement detected by Ventana IHC testing in Chinese patients with early-stage lung adenocarcinoma was $7.9 \%$. ALK rearrangement was significantly associated with younger age, and with the solid predominant with mucin production and invasive mucinous adenocarcinoma subtypes. ALK rearrangement was an adverse prognostic factor in surgicallyresected, early-stage lung adenocarcinoma patients.

\section{MATERIALS AND METHODS}

\section{Patients and specimen characteristics}

Patients with lung adenocarcinoma stages I-III who underwent complete surgical resection and detection of $A L K$ rearrangement at Tianjin Medical University Cancer Institute and Hospital between January 2011 and December 2014 were studied. All patients had an adequate quality and quantity of formalin-fixed paraffin-embedded tissue sections available for analysis. Patients who did not undergo curative resection or had prior histories of cancer or neoadjuvant therapy were excluded. None of the patients in the study cohort had received $A L K$-targeted therapy before tumor recurrence. Data on the patients' clinicopathologic characteristics were retrospectively obtained from the hospital's medical recording system. The study was approved by the Research Ethics Committee of Tianjin Medical University Cancer Institute and Hospital, Tianjin, China.

\section{Clinicopathological evaluation and detection of $A L K$ rearrangement}

Pathologic staging of the patients' NSCLC was based on the 7 th edition of the tumor-node-metastasis (TNM) classification for lung cancer [47]. Histologic subtypes were determined according to the International Association for the Study of Lung Cancer/American Thoracic Society/European Respiratory Society (IASLC/ ATS/ERS) classification of lung adenocarcinoma [36]. $A L K$ rearrangement was detected by the fully-automated Ventana IHC assay using the pre-diluted Ventana antiALK (D5F3) rabbit monoclonal primary antibody, together with the Optiview DAB IHC detection kit and Optiview Amplification kit on the Benchmark XT stainer. Evaluation of the test results was determined by a binary scoring system (positive or negative for $A L K$ status), as previously reported [21]. The patients' $A L K$ rearrangement and histopathologic types were determined by 2 experienced pathologists from Tianjin Lung Cancer Center (Z.Z. and Q.Y.) on a blinded basis.

\section{Assessment of clinical outcomes}

Clinical outcomes were assessed by determining the patients' overall survival (OS), defined as the time from the date of surgical resection to death from any cause; tumorspecific survival (TSS), defined as the time from date of surgical resection to tumor-related death; and disease-free survival (DFS), defined as the time from the date of surgical resection to disease recurrence or death from any cause. If death or recurrence was not observed in any patient, the censoring date was the last day of follow-up.

\section{Statistical analysis}

The prevalence of $A L K$ rearrangement was determined by Ventana IHC testing, and the associations of $A L K$ rearrangement with the patients' clinicopathologic characteristics and treatment outcomes were analyzed using Fisher's exact test (for categorical data) or the Wilcoxon rank-sum test (for continuous data). The DFS, TSS, and OS were plotted using the Kaplan-Meier method and compared by a log-rank test. Multivariate analysis of treatment outcomes was performed using Cox's proportional hazards model. All $P$-values were based on a 2 -sided hypothesis. $P$-values less than 0.05 were considered statistically significant. All analyses were performed using SPSS ${ }^{\circledR}$, version 20.0 (SPSS Inc., Chicago, IL, USA).

\section{Abbreviations}

$A L K$, anaplastic lymphoma kinase; ECOG $P S$, Eastern Cooperative Oncology Group performance status; $E G F R$, epidermal growth factor receptor; $E M L 4$, echinoderm microtubule-associated protein-like 4; HELP, hydrophobic echinoderm microtubule-associated proteinlike protein; FISH, fluorescence in situ hybridization; $H I P 1$, huntingtin-interacting protein $1 ; H R$, hazard ratio; $C I$, confidence interval; IASLC/ATS/ERS, International Association for the Study of Lung Cancer/American Thoracic Society/European Respiratory Society; IHC, immunohistochemistry; $K I F 5 B$, kinesin family member $5 \mathrm{~B} ; K L C 1$, kinesin light chain 1; NCCN, National Comprehensive Cancer Network; NSCLC, non-small cell lung cancer; $R T-P C R$, reverse transcriptase-polymerase chain reaction; $T K I$, tyrosine kinase inhibitor; $T F G$, TRK-fused gene; TNM, tumor-node-metastasis; TPR, translocated promoter region. 


\section{ACKNOWLEDGMENTS}

Editorial assistance with the manuscript was provided by Content Ed Net, Shanghai Co. Ltd.

\section{CONFLICTS OF INTEREST}

None of the authors has any conflicts of interest to declare.

\section{FINANCIAL SUPPORT}

This study was supported by a grant from the General Programs of the National Natural Science Foundation of China (to Chun Huang) [No. 81372467]. The funding source had no involvement in the design of the study, the collection, analysis and interpretation of data, the writing of the report, or the decision to submit it for publication.

\section{REFERENCES}

1. Chen W, Zheng R, Baade PD, Zhang S, Zeng H, Bray F, Jemal A, Yu XQ, He J. Cancer statistics in China, 2015. CA Cancer J Clin. 2016; 66:115-132.

2. Ettinger DS, Bepler G, Bueno R, Chang A, Chang JY, Chirieac LR, D'Amico TA, Demmy TL, Feigenberg SJ, Grannis FW Jr, Jahan T, Jahanzeb M, Kessinger A, et al. Non-small cell lung cancer clinical practice guidelines in oncology. J Natl Compr Canc Netw. 2006; 4:548-582.

3. Lynch TJ, Bell DW, Sordella R, Gurubhagavatula S, Okimoto RA, Brannigan BW, Harris PL, Haserlat SM, Supko JG, Haluska FG, Louis DN, Christiani DC, Settleman $\mathrm{J}$, et al. Activating mutations in the epidermal growth factor receptor underlying responsiveness of non-small-cell lung cancer to gefitinib. N Engl J Med. 2004; 350:2129-2139.

4. Paez JG, Janne PA, Lee JC, Tracy S, Greulich H, Gabriel S, Herman P, Kaye FJ, Lindeman N, Boggon TJ, Naoki K, Sasaki H, Fujii Y, et al. EGFR mutations in lung cancer: correlation with clinical response to gefitinib therapy. Science. 2004; 304:1497-1500.

5. Soda M, Choi YL, Enomoto M, Takada S, Yamashita Y, Ishikawa S, Fujiwara S, Watanabe H, Kurashina K, Hatanaka H, Bando M, Ohno S, Ishikawa Y, et al. Identification of the transforming EML4-ALK fusion gene in non-small-cell lung cancer. Nature. 2007; 448:561-566.

6. Soda M, Takada S, Takeuchi K, Choi YL, Enomoto M, Ueno T, Haruta H, Hamada T, Yamashita Y, Ishikawa Y, Sugiyama Y, Mano H. A mouse model for EML4-ALKpositive lung cancer. Proc Natl Acad Sci U S A. 2008; 105:19893-19897.

7. Koivunen JP, Mermel C, Zejnullahu K, Murphy C, Lifshits E, Holmes AJ, Choi HG, Kim J, Chiang D, Thomas R, Lee J, Richards WG, Sugarbaker DJ, et al. EML4-ALK fusion gene and efficacy of an ALK kinase inhibitor in lung cancer. Clin Cancer Res. 2008; 14:4275-4283.

8. Choi YL, Takeuchi K, Soda M, Inamura K, Togashi Y, Hatano S, Enomoto M, Hamada T, Haruta H, Watanabe H, Kurashina K, Hatanaka H, Ueno T, et al. Identification of novel isoforms of the EML4-ALK transforming gene in non-small cell lung cancer. Cancer Res. 2008; 68:4971-4976.

9. Rikova K, Guo A, Zeng Q, Possemato A, Yu J, Haack H, Nardone J, Lee K, Reeves C, Li Y, Hu Y, Tan Z, Stokes $M$, et al. Global survey of phosphotyrosine signaling identifies oncogenic kinases in lung cancer. Cell. 2007; 131:1190-1203.

10. Takeuchi K, Choi YL, Togashi Y, Soda M, Hatano S, Inamura K, Takada S, Ueno T, Yamashita Y, Satoh Y, Okumura S, Nakagawa K, Ishikawa Y, et al. KIF5BALK, a novel fusion oncokinase identified by an immunohistochemistry-based diagnostic system for ALKpositive lung cancer. Clin Cancer Res. 2009; 15:3143-3149.

11. Togashi Y, Soda M, Sakata S, Sugawara E, Hatano S, Asaka R, Nakajima T, Mano H, Takeuchi K. KLC1-ALK: a novel fusion in lung cancer identified using a formalin-fixed paraffin-embedded tissue only. PLoS One. 2012; 7:e31323.

12. Hong M, Kim RN, Song JY, Choi SJ, Oh E, Lira ME, Mao M, Takeuchi K, Han J, Kim J, Choi YL. HIP1-ALK, a novel fusion protein identified in lung adenocarcinoma. J Thorac Oncol. 2014; 9:419-422.

13. Choi YL, Lira ME, Hong M, Kim RN, Choi SJ, Song JY, Pandy K, Mann DL, Stahl JA, Peckham HE, Zheng Z, Han J, Mao M, et al. A novel fusion of TPR and ALK in lung adenocarcinoma. J Thorac Oncol. 2014; 9:563-566.

14. Kim RN, Choi YL, Lee MS, Lira ME, Mao M, Mann D, Stahl J, Licon A, Choi SJ, Van Vrancken M, Han J, Wlodarska I, Kim J. SEC31A-ALK fusion gene in lung adenocarcinoma. Cancer Res Treat. 2016; 48:398-402.

15. Selinger CI, Rogers TM, Russell PA, O'Toole S, Yip P, Wright GM, Wainer Z, Horvath LG, Boyer M, McCaughan B, Kohonen-Corish MR, Fox S, Cooper WA, et al. Testing for ALK rearrangement in lung adenocarcinoma: a multicenter comparison of immunohistochemistry and fluorescent in situ hybridization. Mod Pathol. 2013; 26:1545-1553.

16. Zhou J, Zhao J, Sun K, Wang B, Wang L, Chen X, Zheng J, You Q, Wang X, Ding W, Zhou J. Accurate and economical detection of ALK positive lung adenocarcinoma with semiquantitative immunohistochemical screening. PLoS One. 2014; 9:e92828.

17. Rodig SJ, Mino-Kenudson M, Dacic S, Yeap BY, Shaw A, Barletta JA, Stubbs H, Law K, Lindeman N, Mark E, Janne PA, Lynch T, Johnson BE, et al. Unique clinicopathologic features characterize ALK-rearranged lung adenocarcinoma in the western population. Clin Cancer Res. 2009; 15:5216-5223.

18. Shen Q, Wang X, Yu B, Shi S, Liu B, Wang Y, Xia Q, Rao Q, Zhou X. Comparing four different ALK antibodies with 
manual immunohistochemistry (IHC) to screen for ALKrearranged non-small cell lung cancer (NSCLC). Lung Cancer. 2015; 90:492-498.

19. Wynes MW, Sholl LM, Dietel M, Schuuring E, Tsao MS, Yatabe Y, Tubbs RR, Hirsch FR. An international interpretation study using the ALK IHC antibody D5F3 and a sensitive detection kit demonstrates high concordance between ALK IHC and ALK FISH and between evaluators. J Thorac Oncol. 2014; 9:631-638.

20. von Laffert M, Warth A, Penzel R, Schirmacher P, Kerr KM, Elmberger G, Schildhaus HU, Büttner R, Lopez-Rios F, Reu S, Kirchner T, Pauwels P, Specht K, et al. Multicenter immunohistochemical ALK-testing of non-small-cell lung cancer shows high concordance after harmonization of techniques and interpretation criteria. J Thorac Oncol. 2014; 9:1685-1692.

21. Ying J, Guo L, Qiu T, Shan L, Ling Y, Liu X, Lu N. Diagnostic value of a novel fully automated immunochemistry assay for detection of ALK rearrangement in primary lung adenocarcinoma. Ann Oncol. 2013; 24:2589-2593.

22. Shaw AT, Yeap BY, Mino-Kenudson M, Digumarthy SR, Costa DB, Heist RS, Solomon B, Stubbs H, Admane S, McDermott U, Settleman J, Kobayashi S, Mark EJ, et al. Clinical features and outcome of patients with non-smallcell lung cancer who harbor EML4-ALK. J Clin Oncol. 2009; 27:4247-4253.

23. Kim HR, Shim HS, Chung JH, Lee YJ, Hong YK, Rha SY, Kim SH, Ha SJ, Kim SK, Chung KY, Soo R, Kim JH, Cho BC. Distinct clinical features and outcomes in neversmokers with nonsmall cell lung cancer who harbor EGFR or KRAS mutations or ALK rearrangement. Cancer. 2012; 118:729-739.

24. Zhou JX, Yang H, Deng Q, Gu X, He P, Lin Y, Zhao M, Jiang J, Chen H, Lin Y, Yin W, Mo L, He J. Oncogenic driver mutations in patients with non-small-cell lung cancer at various clinical stages. Ann Oncol. 2013; 24:1319-1325.

25. Warth A, Penzel R, Lindenmaier H, Brandt R, Stenzinger A, Herpel E, Goeppert B, Thomas M, Herth FJ, Dienemann H, Schnabel PA, Schirmacher P, Hoffmann $\mathrm{H}$, et al. EGFR, KRAS, BRAF and ALK gene alterations in lung adenocarcinomas: patient outcome, interplay with morphology and immunophenotype. Eur Respir J. 2014; 43:872-883.

26. Takeda M, Okamoto I, Sakai K, Kawakami H, Nishio K, Nakagawa K. Clinical outcome for EML4-ALK-positive patients with advanced non-small-cell lung cancer treated with first-line platinum-based chemotherapy. Ann Oncol. 2012; 23:2931-2936.

27. Lee JK, Park HS, Kim DW, Kulig K, Kim TM, Lee SH, Jeon YK, Chung DH, Heo DS, Kim WH, Bang YJ. Comparative analyses of overall survival in patients with anaplastic lymphoma kinase-positive and matched wildtype advanced nonsmall cell lung cancer. Cancer. 2012; 118:3579-3586.
28. Paik JH, Choi CM, Kim H, Jang SJ, Choe G, Kim DK, Kim HJ, Yoon H, Lee CT, Jheon S, Choe JY, Chung JH. Clinicopathologic implication of ALK rearrangement in surgically resected lung cancer: a proposal of diagnostic algorithm for ALK-rearranged adenocarcinoma. Lung Cancer. 2012; 76:403-409.

29. Kim MH, Shim HS, Kang DR, Jung JY, Lee CY, Kim DJ, Lee JG, Bae MK, Kim HR, Lim SM, Kim EY, Park JS, Chung KY, et al. Clinical and prognostic implications of ALK and ROS1 rearrangements in never-smokers with surgically resected lung adenocarcinoma. Lung Cancer. 2014; 83:389-395.

30. Blackhall FH, Peters S, Bubendorf L, Dafni U, Kerr KM, Hager H, Soltermann A, O’Byrne KJ, Dooms C, Sejda A, Hernández-Losa J, Marchetti A, Savic S, et al. Prevalence and clinical outcomes for patients with ALK-positive resected stage I to III adenocarcinoma: results from the European Thoracic Oncology Platform Lungscape Project. J Clin Oncol. 2014; 32:2780-2787.

31. Tantraworasin A, Lertprasertsuke N, Kongkarnka S, Euathrongchit J, Wannasopha Y, Saeteng S. Retrospective study of ALK rearrangement and clinicopathological implications in completely resected non-small cell lung cancer patients in Northern Thailand: role of screening with D5F3 antibodies. Asian Pac J Cancer Prev. 2014; 15:3057-3063.

32. Ohba T, Toyokawa G, Osoegawa A, Hirai F, Yamaguchi M, Taguchi K, Seto T, Takenoyama M, Ichinose Y, Sugio K. Mutations of the EGFR, K-ras, EML4-ALK, and BRAF genes in resected pathological stage I lung adenocarcinoma. Surg Today. 2016; 46:1091-1098.

33. Wu SG, Kuo YW, Chang YL, Shih JY, Chen YH, Tsai MF, Yu CJ, Yang CH, Yang PC. EML4-ALK translocation predicts better outcome in lung adenocarcinoma patients with wild-type EGFR. J Thorac Oncol. 2012; 7:98-104.

34. Shaw AT, Kim DW, Nakagawa K, Seto T, Crino L, Ahn MJ, De Pas T, Besse B, Solomon BJ, Blackhall F, Wu YL, Thomas M, O’Byrne KJ, et al. Crizotinib versus chemotherapy in advanced ALK-positive lung cancer. N Engl J Med. 2013; 368:2385-2394.

35. Solomon BJ, Mok T, Kim DW, Wu YL, Nakagawa K, Mekhail T, Felip E, Cappuzzo F, Paolini J, Usari T, Iyer S, Reisman A, Wilner KD, et al. First-line crizotinib versus chemotherapy in ALK-positive lung cancer. N Engl J Med. 2014; 371:2167-2177.

36. Travis WD, Brambilla E, Van Schil P, Scagliotti GV, Huber RM, Sculier JP, Vansteenkiste J, Nicholson AG. Paradigm shifts in lung cancer as defined in the new IASLC/ATS/ERS lung adenocarcinoma classification. Eur Respir J. 2011; 38:239-243.

37. Possidente L, Landriscina M, Patitucci G, Borgia L, Lalinga V, Vita G. ALK rearrangement in specific subtypes of lung adenocarcinoma: immunophenotypic and morphological features. Med Oncol. 2017; 34:76. 
38. Dong YJ, Cai YR, Zhou LJ, Su D, Mu J, Chen XJ, Zhang LI. Association between the histological subtype of lung adenocarcinoma, EGFR/KRAS mutation status and the ALK rearrangement according to the novel IASLC/ATS/ ERS classification. Oncol Lett. 2016; 11:2552-2558.

39. Yu Y, Ding Z, Zhu L, Teng H, Lu S. Frequencies of ALK rearrangements in lung adenocarcinoma subtypes: a study of 2299 Chinese cases. Springerplus. 2016; 5:894.

40. Zhao F, Xu M, Lei H, Zhou Z, Wang L, Li P, Zhao J, Hu P. Clinicopathological characteristics of patients with nonsmall-cell lung cancer who harbor EML4-ALK fusion gene: a meta-analysis. PLoS One. 2015; 10:e0117333.

41. von der Thusen JH, Tham YS, Pattenden H, Rice A, Dusmet M, Lim E, Nicholson AG. Prognostic significance of predominant histologic pattern and nuclear grade in resected adenocarcinoma of the lung: potential parameters for a grading system. J Thorac Oncol. 2013; 8:37-44.

42. Makinen JM, Laitakari K, Johnson S, Makitaro R, Bloigu R, Lappi-Blanco E, Kaarteenaho R. Nonpredominant lepidic pattern correlates with better outcome in invasive lung adenocarcinoma. Lung Cancer. 2015; 90:568-574.

43. Murakami S, Ito H, Tsubokawa N, Mimae T, Sasada S, Yoshiya T, Miyata Y, Yokose T, Okada M, Nakayama H. Prognostic value of the new IASLC/ATS/ERS classification of clinical stage IA lung adenocarcinoma. Lung Cancer. 2015; 90:199-204.
44. Yoshiya T, Mimae T, Tsutani Y, Tsubokawa N, Sasada S, Miyata Y, Kushitani K, Takeshima Y, Murakami S, Ito H, Nakayama H, Okada M. Prognostic role of subtype classification in small-sized pathologic N0 invasive lung adenocarcinoma. Ann Thorac Surg. 2016; 102:1668-1673.

45. Zhao Y, Wang R, Shen X, Pan Y, Cheng C, Li Y, Shen L, Zhang Y, Li H, Zheng D, Ye T, Zheng S, Sun Y, et al. Minor components of micropapillary and solid subtypes in lung adenocarcinoma are predictors of lymph node metastasis and poor prognosis. Ann Surg Oncol. 2016; 23:2099-2105.

46. Luo J, Wang R, Han B, Zhang J, Zhao H, Fang W, Luo Q, Yang J, Yang Y, Zhu L, Chen T, Cheng X, Huang Q, et al. Solid predominant histologic subtype and early recurrence predict poor postrecurrence survival in patients with stage I lung adenocarcinoma. Oncotarget. 2017; 8:7050-7058. https://doi.org/10.18632/oncotarget.12540.

47. Goldstraw P, Crowley J, Chansky K, Giroux DJ, Groome PA, Rami-Porta R, Postmus PE, Rusch V, Sobin L; International Association for the Study of Lung Cancer International Staging Committee; Participating Institutions. The IASLC Lung Cancer Staging Project: proposals for the revision of the TNM stage groupings in the forthcoming (seventh) edition of the TNM classification of malignant tumours. J Thorac Oncol. 2007; 2:706-714. 